This item was submitted to Loughborough's Research Repository by the author.

Items in Figshare are protected by copyright, with all rights reserved, unless otherwise indicated.

\title{
Study of the resilience of nuclear power plants in response to climate change
}

PLEASE CITE THE PUBLISHED VERSION

https://doi.org/10.3850/978-981-18-2016-8_110-cd

PUBLISHER

Research Publishing Services

VERSION

VoR (Version of Record)

PUBLISHER STATEMENT

Reproduced with permission of the author

LICENCE

CC BY-NC-ND 4.0

\section{REPOSITORY RECORD}

Yan, Rundong (Derek), and Sarah Dunnett. 2021. "Study of the Resilience of Nuclear Power Plants in Response to Climate Change”. Loughborough University. https://hdl.handle.net/2134/16740298.v1. 


\title{
Study of the Resilience of Nuclear Power Plants in Response to Climate Change
}

\author{
Rundong Yan; Sarah Dunnett \\ Department of Aeronautical and Automotive Engineering, Loughborough University, Loughborough, Leicestershire, LE11 $3 T U$, UK. \\ E-mail: r.yan@lboro.ac.uk \\ E-mail: s.j.dunnett@lboro.ac.uk
}

\begin{abstract}
In recent years, it has become even more challenging to ensure the safety of nuclear power plants due to accelerated climate change. This is because some existing safety systems in the plants are not able to cope with new issues introduced or aggravated by climate change. In response to this need, this paper will analyze the present related reactor safety systems and propose and discuss a measure that can potentially improve the resilience of the reactor system to climate change. To facilitate the research, the intake structure blockage caused by the outbreak of a kind of marine organism whose size varies from $4 \mathrm{~mm}$ to $40 \mathrm{~mm}$, is chosen as a case study. The study will consider the ability of the system to anticipate for the events, absorb the impact of the events to the system, and recover from perturbations. To facilitate the research, a mathematical model will be developed using Petri nets to simulate the reliability and health states of the related safety systems, the occurrence of disruptive events, the corresponding responses of the nuclear system, and the possible operation states and recovery of the system from the disruptive events. The results indicated that the intake structure blockage caused by such external events cannot be ignored. The research is expected lay a solid foundation for future nuclear power system design and the resilience assessment of nuclear reactor systems.
\end{abstract}

Keywords: Resilience, Nuclear power plant, Petri nets, Mathematical modeling, Simulation, Climate change.

\section{Introduction}

The safety and resilience of nuclear power has consistently been a concern to the public and scientific community due to the potentially catastrophic consequences of nuclear accidents. After the Fukushima nuclear accident in 2011, people have further realized that the risk external events could bring to the nuclear power plants (NPPs) can be much more catastrophic than expected. Climate change is predicted to have a significant impact on the safety and resilience of power systems (Panteli et al. 2016). The interaction between NPPs and the surrounding environment has been a hot topic of research since the construction of the first commercial NPP. Recent climate change has brought further safety challenges to NPPs, which include the extreme high or low temperature of the service water, drought, flood, and facility damage caused by hurricanes or tsunamis, etc. Among all these issues, the impact of abnormal flora and fauna levels due to climate change on the safety and resilience of NPP has not received much attention by the relevant utilities.

The intake structure blockage of the NPPs on the coasts caused by the outbreak or excessive multiplication of some organisms, also known as biological fouling, is a typical example. There are 179 reactors that used seawater for cooling in 2011 (Tang et al. 2017). From 1980 to 1990, at least 10 NPPs reported loss of service water because of biological fouling (mussels, fish, shrimps, jellyfish, etc.) around the world (IAEA 2003). 61 intake blockage events, where most of them are due to the marine organisms, have been reported between 2004 and 2008 according to the Institute of Nuclear Power Research (Tang et al. 2017).
Around $80 \%$ of these events lead to a reduction of power generation, or even the reactor shutdown. In China only, 29 sea creature events were reported from 5 operating NPPs from 2014 to 2017 where 8 of them caused blockage (Wang and Duan 2017). From this statistical data, it is found that these events are becoming more frequent and they could happen in every type of NPP. There is limited research about the impact of biological fouling on the safety of nuclear reactors. Fu et al. found that there are four critical factors which could significantly affect the possibility of the cooling water intake blockage of nuclear power plants $(\mathrm{Fu}$ et al. 2020). They are hydrodynamic conditions, the outbreak timing of organisms, design of the filter systems, and characteristics of species.

In order to investigate the, the impact of such events on the safety and resilience of NPP, this paper aims to develop a mathematical model using the Petri net (PN) approach to simulate the reliability and health states of the related safety systems, the occurrence of marine organism induced intake blockage events, the corresponding responses of the nuclear system, and the possible operation states and recovery of the system from the disruptive events. A typical pressurized heavy-water reactor (PHWR) design is chosen for analysis.

\section{System Description}

The demonstration reactor system used in this work is a typical PHWR located on a coast. Fig. 1 is a schematic diagram showing the layout of the intake structure of the 
circulating water system with its filtration system, which is developed based on (Fu et al. 2020; Turnpenny et al. 2010).

Seawater flows through the filtering grids to prevent the debris and biota from getting into the cooling system first. Then, it is pumped via two circulating water pumps to remove redundant heat generated in the reactor system. Finally, the heated water flows back to the sea. The filtration system for the water intake is composed of three parts. The first part is a coarse grid with filter size $200 \mathrm{~mm}$, the second is a fine grid with filter size $50 \mathrm{~mm}$, and the third part is a rotating filter with filter size $3 \mathrm{~mm}$. These grids are designed to filter out the debris and biota with different sizes.

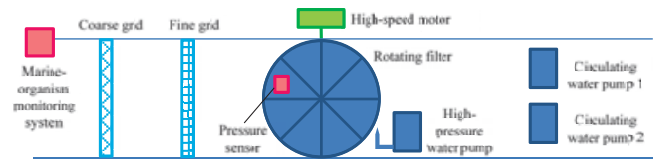

Fig. 1. A schematic of the intake structure of the circulating water system

There are a number of different marine organisms which could cause the intake blockages. For example, a British advanced gas-cooled reactor was shut down by a massive jellyfish influx in 1991 (Turnpenny et al. 2010). The reactors at the United Kingdom's Torness Nuclear Power Station in UK were shut down because of seaweed in 2010, 2013, 2016, and 2018 (Turnpenny et al. 2010; Edwards 2013; BBC 2016; Vaughan 2018). In 2011, Salem Units $1 \& 2$ had to reduce their power due to the intake blockage by large wood pieces bought in by flood (USNRC 2011). In this research, the possible intake blockage due to the outbreak of a kind of acetes (a genus of small shrimp) whose size varies from $4 \mathrm{~mm}$ to $40 \mathrm{~mm}$ is considered. They migrate to the sea region near the coastline around the middle of March for breeding every year. Their spawning of the population reaches peak at $20^{\circ} \mathrm{C}$ (Oh and Jeong 2003). Then, as the temperature in the far sea increases, they will start to move away from the coast.

In order to mitigate the impact of these external events, several safety systems are installed in the reactor system. The marine-organism monitoring system located at the intake entrance is used to detect the biology explosion and to provide early warnings (Tang et al. 2017). The system is able to detect a fan-shape range with a radius of 60 meters. Once the operators are alerted by the system, they will then judge the necessity for sending out the emergency fishing team to remove the acetes based on the situation. As the size of these organisms are small, they can only be filtered out by the rotating filter. Hence, only the safety systems designed for the third part of the filtration system are considered in the research. As the marine organisms build up on the filter, the pressure applied on it will increase gradually, which can be measured by the pressure sensor. If the pressure reaches a certain threshold, the high-speed motor can be activated to increase the rotating speed of the rotating filter. If the pressure still increases, the highpressure water pump can eject the water on the filter to wash off anything on it. It is assumed that both systems are able to reduce the pressure on the filter with a rate of $3 \mathrm{~Pa}$ per second. When the pressure reaches the trip threshold, one water circulating pump will trip which means the power output of the reactor will need to be reduced to $50 \%$. This will also reduce the pressure on the filter. However, if the pressure increases to the trip threshold again, another pump will also trip. Then, the reactor must be shut down immediately. Two independent and diverse, fast-acting safety shutdown systems are in place: the primary system (SDS1) relies on the use of neutron-absorbing shutoff rods suspended above the reactor by electromagnets; the secondary system (SDS2) is designed to inject highpressure gadolinium nitrate into the moderator. The mentioned pressure thresholds for different system responses are listed in Table 1. It should be noted that other related safety systems such as the auxiliary cooling system and backup cooling sink will not be considered in the study but will be studied in future work.

Table 1. Pressure thresholds for different system responses

\begin{tabular}{ll}
\hline System responses & $\begin{array}{l}\text { Pressure thresholds } \\
(\mathrm{kPa})\end{array}$ \\
\hline $\begin{array}{l}\text { Activation of high- } \\
\text { speed motor }\end{array}$ & 1.96 \\
$\begin{array}{l}\text { Activation of high- } \\
\text { pressure water pump }\end{array}$ & 2.94 \\
Pump trip & 4.90 \\
\hline
\end{tabular}

It is assumed that the failure of these subsystems follows either Weibull or exponential distributions. The distribution parameters including the shape parameter $(\beta)$ characteristic life $(\eta)$, and failure rate $(\lambda)$ are listed in Table 2 . This information is derived based on historical failure data (IAEA 1988; Bian et al. 2009).

Table 2. Failure and repair data of the subsystems in the reactor

\begin{tabular}{lll}
\hline Subsystems & Distribution & Parameters \\
\hline $\begin{array}{l}\text { Marine-organism } \\
\text { monitoring system }\end{array}$ & Exponential & $\lambda=3.84 \times 10^{-5} /$ day \\
$\begin{array}{l}\text { Pressure sensor } \\
\text { High-speed motor }\end{array}$ & Exponential & $\lambda=5.10 \times 10^{-3} /$ day \\
Exponential & $\lambda=3.00 \times 10^{-4} /$ day \\
pump & Exponential & $\lambda=4.00 \times 10^{-3} /$ day \\
SDS1 & Weibull & $\beta=1.3000$ \\
& & $\eta=656.89$ years \\
SDS2 & Weibull & $\beta=1.5320$ \\
& & $\eta=5.70$ years \\
\hline
\end{tabular}

\section{Methodology for Modelling}

A PN is a direct bipartite graph that consists of three types of symbols, namely circles, rectangles, and arrows as illustrated in Fig. 2. Circles represent the places, which are conditions or states of a system. The condition place, filled light gray in the figure, means the model will perform predefined actions if the conditions are met in the place. In addition, the place filled dark gray means the simulation 
will end if a token is placed in one of these places. Rectangles represent the transitions, which are actions or events causing the change of condition or state. If the time of the transition is zero, the rectangle will be filled black, otherwise it is empty. Arrows, known as arcs in PNs, link places and transitions together. Arcs with a slash on and a number, $n$, next to the slash represent a combination of $n$ single arcs and the arc is said to have a weight $n$. A transition can be enabled if the number of tokens in every input place is greater than or equal to the corresponding weights of the arcs to the transition. Once a transition is enabled it will fire after the time associated with it and tokens will be taken out of the input places and put into the output places. In addition, the dashed arrows mean the link between the connected places and transitions are conditional. After a transition is enabled, the probability of the expected tokens being produced in the output places connected by a conditional arc is predefined. In addition, an arc with a small circle on one end is known as an inhibitor arc. This is able to prevent a transition from firing when enabled. Finally, small black filled circles represent tokens that carry the information in the PNs. The movement of the tokens between the places in the net gives the dynamic properties of PNs. The positions of the tokens in a PN at a given time shows the state of the system being modelled at that time.

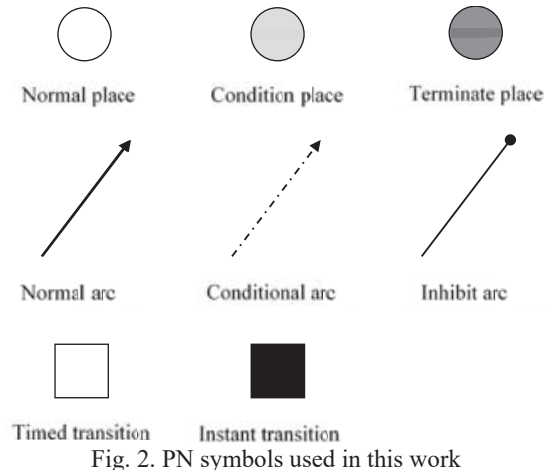

To facilitate the research, three PNs are constructed to model the states of the reactor system, the accidents or hazards and the corresponding consequence, and more importantly the responses to the accidents and recovery from the accidents. These are:

- Reactor System Petri Net (RSPN) - simulates the occurrence of the external events and describes the working and failure states of the safety systems in the reactor.

- $\quad$ Detection and Response Petri Net (DRPN) - simulates the detection of the external events and the mitigation processes for maintaining the safety and operation of the reactor system.

- $\quad$ Shutdown Petri Net (SDPN)- simulates the shutdown process of the reactor system.

These three PNs are linked together and share information with each other as illustrated in Fig. 3. The external events and the health states of safety systems in the reactor will be simulated in the RSPN. The responses of the reactor system after the occurrence of the external events will be simulated in the DRPN and SDPN. It should be noted the responses will be different due to the availability of the safety systems and the magnitude of the external events. Therefore, the times of some key transitions such as those representing the time for fault detection and activating the safety systems, will be computed accordingly. The details of the nets are discussed in the following sections.

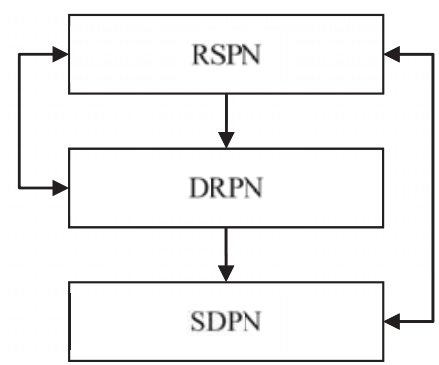

Fig. 3. The overall structure of the model

\section{Model Construction}

\subsection{Reactor System Petri Net (RSPN)}

The RSPN can be divided into three parts as illustrated in Fig. 4, Fig. 5, and Fig. 6, respectively. The first part simulates the health states of the reactor subsystems considered in the research, which include two independent shutdown systems, a marine-organism monitoring system, a pressure sensor, a high-speed motor, and a high-pressure water pump. The PN for the high-pressure water pump is shown in Fig. 4 as an example. In this research, only two health states for the systems are considered, namely normal (UP) and failed (DOWN). More complex and detailed system health states can be included in the model and this will be considered in the future. The net shown represents the pump is in its 'healthy' state as a token is in the 'UP' place. The timed transition 'IS1' between these two places represents the natural degradation process of the pump. The time for the failure transitions can be computed by using random sampling and the failure data given in Table 2.
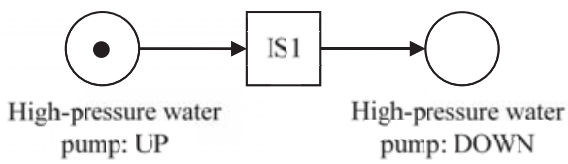

Fig. 4. RSPN part 1: natural degradation of the high-pressure water pump

The second part shown in Fig. 5 is developed to model the occurrence of the external events. The external events considered in the study is the outbreak of the acetes. It is assumed that the earliest date this event could happen is $1^{\text {st }}$ February and it is most likely to happen around the middle of March. Also, the nuclear reactor initiates its operation on 
$1^{\text {st }}$ October. Hence, the time delay of the ' $\mathrm{D} 1$ ' transition is the time from the initiation of the power generation to when the first outbreak event could occur, which is 123 days. Once 'D1' is fired, a token will be produced in both the places, 'External event might occur' and 'Start of annual count'. The token produced in the former place will enable the transition, 'D3', which aims to simulate the exact time the external event is going to occur. Its transition time is assumed to follow a Normal distribution with the mean $(\mu)$ of 44.625 days and the standard deviation $(\sigma)$ of 20 days. It is worth mentioning that the time is set to be no less than 0 and no larger than 89.25 days. The transition is connected to four output condition places representing the external events with different severities by the conditional arcs. It means that only one token will be produced in either of these four places. The severity of the external event in the study is deduced based on the total population and population density of acetes in the area near the intake of the nuclear reactor system. The features of the external events with different severity levels are listed in Table 3. The conditions defined for these places are that if a token is produced in either of these places, the produced token will be removed from the net and the DRPN will be embedded into simulation. Moreover, a token produced in the place, 'Start of annual count', will enable the Transition 'D2' whose duration is set to be one year, so that the external event will be generated every year until the end of the reactor's designed lifetime, which is set to be 30 years.

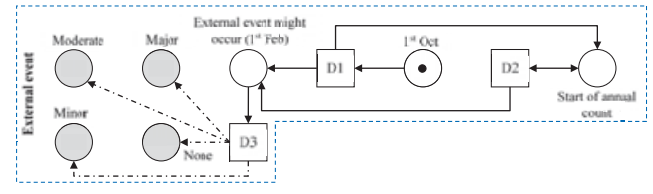

Fig. 5. RSPN part 2: generation of external events

Table 3. Features of the extern events

\begin{tabular}{lll}
\hline Severity levels & $\begin{array}{l}\text { Duration } \\
\text { (minutes) }\end{array}$ & $\begin{array}{l}\text { Pressure increase rate on } \\
\text { the rotating filter }(\mathrm{Pa} / \mathrm{s})\end{array}$ \\
\hline None & 0 & 0 \\
Minor & 15 & 3 \\
Moderate & 30 & 6 \\
Major & 60 & 15 \\
\hline
\end{tabular}

The final part of the RSPN shown in Fig. 6 simulates the periodic maintenance of the reactor system. All the subsystems considered will be regularly inspected and repaired. It is assumed that the systems will be restored to their 'UP' states after each periodic maintenance. The 'PM1' transition is the time interval between periodic maintenance events. The 'PM2' transition is the time required for the inspection and repair of the whole system, which is set two be 2 days. Once there is a token produced in the 'PM completed' Place, all the transition times will be recomputed except 'D2' and 'D3' for simulating the time for the next external event.

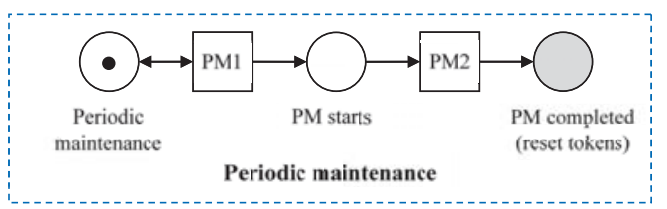

Fig. 6. RSPN part 3: periodic maintenance

\subsection{Detection and Response Petri Net (DRPN)}

The DRPN shown in Fig. 7 simulates the responses of the reactor system to the external event. The token in the 'Reach the monitoring region' place means the fauna has entered the region which can be detected by the marine-organism monitoring system. The health states of the related safety system modelled in the RSPN will be fed to the DRPN. For example, if a token is in the 'monitoring system: UP' place in the RSPN, a token will also be given to the place with the same label in the DRPN correspondingly. If the monitoring system is in 'UP' state, the 'MS1' transition will fire immediately. This will then be fed to the PN module simulating the responses of the operators and the emergency fishing team, which is represented by a dashed box in the figure. Once the operators are alerted, they will judge the scenario first. If the event is 'Major', the operators will call the emergency fishing team to remove the acetes from the area. It is assumed that the operators can always judge it correctly. The time for the emergency team to reach the fishing area depends on various factors such as the distance to the area, the type of fishing vessel, the weather conditions and so on. In the research, it is assumed to follow a Normal distribution with the mean $(\mu)$ of 45 minutes and the standard deviation $(\sigma)$ of 10 minutes. The time required to remove the acetes is assumed to be 10 minutes, which is modelled via the transition, 'MS5'. Once the fishing mission is completed, a token will be produced in the 'Event past' place, which means the event has ended. If this is not the case, the event can be regarded as ended after the firing of the 'E1' transition. The time of the transition depends on the severity of the external event. Once the event is past, the DRPN will be removed from the simulation.

Alongside the actions of the operators and the fishing team, the reactor system will automatically respond to the external event. It is assumed that the seawater flow rate is $0.3 \mathrm{~m} / \mathrm{s}$ and the distance between the rotating filter and the furthest monitoring range is assumed to be 80 meters. In addition, the swimming ability of acetes is limited. Hence, the time of the ' $\mathrm{S} 1$ ' transition can be calculated, which is 266.7 seconds. The 'S2' transition represents the time until the pressure on the filter is increased to the threshold for the activation of the fast-rotating motor. If the pressure sensor is working properly represented by a token in the 'Pressure sensor: UP' place, the reactor system will try to initiate the fast-rotating mode of the filter. Otherwise, the pressure will keep increasing until the shutdown of the reactor or the end of the event. If the abnormal pressure is successfully detected and the fast-rotating motor is 'healthy', the fastrotating mode will be started. However, if the motor is not available or the pressure still keeps increasing to another threshold after the activation as modelled by the transitions, 
'S7' and 'S8', respectively, the high-pressure water pump will be activated to wash off the acetes on the filter. The activation process is modelled in the PN module represented by the dashed box, which is labelled as 'Activation of the high-pressure water pump'. If these two measures are still insufficient to control the pressure applied on the filter, the pressure will keep increasing until one pump, or both pumps, are tripped which means the reactor needs to be shut down immediately. Once a token is produced in the 'Shutdown' place, the DRPN will be removed from the simulation while the SDPN will be imported into it.

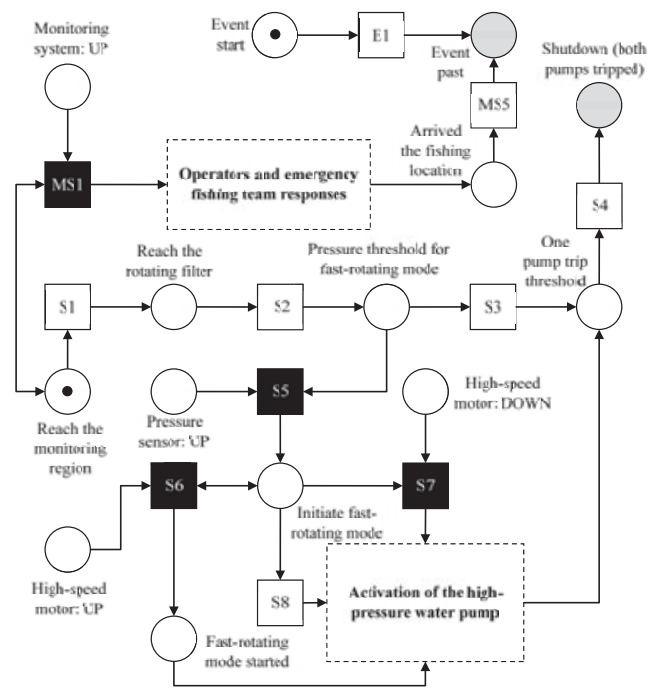

Fig. 7. DRPN

\subsection{Shutdown Petri Net (SDPN)}

The SDPN shown in Fig. 8 is constructed in a similar manner to the DRPN. Once both the circulating water pumps are tripped, the shutoff rods (SDS1) will be activated. If SDS1 fails, the poison injection system (SDS2) will be activated as simulated by the transition, 'SD3'. However, if both shutdown systems fail, a token will be produced in the 'Failed shutdown' place. This means that the reactor cannot be shut down successfully, which will cause rapid melt of the core as modelled by the 'SD6' transition whose time is assumed to be 30 minutes. In this case, the operators need to activate the emergency plan to manage the crisis before the core is melt. This is the worstcase considered in this study. Since it is assumed that the melted reactor core is not recoverable, the simulation will be ended immediately once a token is produced in the terminate place 'Core melting'. If the reactor is shut down successfully, i.e. a token is produced in the 'Successful shutdown' place, the reactor can be regarded as safe. It is assumed that the time of the 'SD7' transition is the restoration time of the reactor's performance required, which is set to be 48 hours, it is assumed that all the related subsystems will be inspected and repaired before the restoration. Hence, the health state of the subsystem modelled in the RSPN will be restored to 'UP' and their time to the next failure will be recomputed. It should be noted that the SDPN will be removed from the simulation.

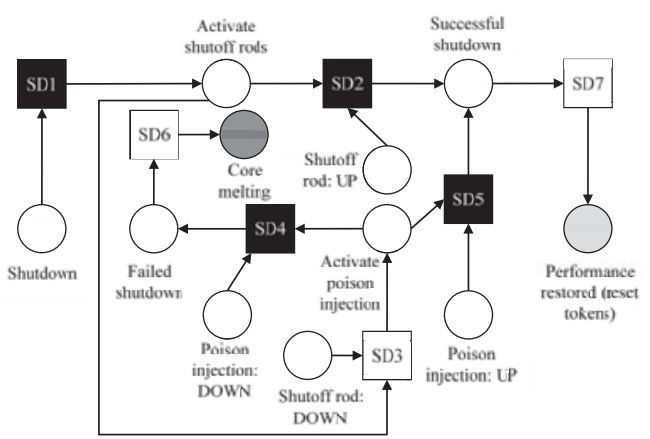

Fig. 8. SDPN

\section{Simulation}

In order to evaluate the resilience of the reactor system, the PN models proposed in the previous section can be used for simulation. The system information in Table 1, the failure data in Table 2, the features of the external events in Table 3 , and other related defined data such as the detection range of the monitoring system are used as inputs to the simulation. The following are the simulation assumptions:

- Seawater flow rate is constant.

- Periodic maintenance is conducted every 2 years.

- The auxiliary cooling systems are always available after the shutdown of the reactor.

- The probabilities of the occurrence of the external event are constant within the lifetime of the reactor.

\subsection{Simulation results}

In order to investigate the impact of the external events, which can be affected by climate change, the simulations are conducted for two sets of the predefined occurrence probabilities of the external events listed in Table 4 for comparison. The first set (Set 1) is assumed to be the normal scenario and the second one (Set 2) is derived based on the assumption that the temperature of the seawater in the coast area is more like to be higher than usual due to climate change. One million simulation iterations are conducted for each set to ensure the convergence of the results.

Table 4. Occurance probilbity of the extenal events

\begin{tabular}{lll}
\hline Severity levels & Set 1 & Set 2 \\
\hline None & 0.6000 & 0.5000 \\
Minor & 0.2500 & 0.3125 \\
Moderate & 0.1000 & 0.1250 \\
Major & 0.0500 & 0.0625
\end{tabular}

The simulation results are listed in Table 5. From the table, it is found that the occurrence probabilities of all the events are increased by about $25 \%$. Similarly, the numbers 
of the reactor shutdown and reduced performance due to the external events are also increased by about $25 \%$. This suggests climate change does affect the operation of the reactor system.

Table 5. Simulation results for normal scenario (Set 1) and when affected by climate change (Set 2)

\begin{tabular}{llllllll}
\hline Sets & $N_{1}$ & $N_{2}$ & $N_{3}$ & $N_{r}$ & $N_{s}$ & $N_{m}$ & $P$ \\
\hline Set 1 & 7.50 & 3.00 & 1.50 & 2.67 & 1.49 & $<10^{-4}$ & 0.65 \\
Set 2 & 9.37 & 3.75 & 1.87 & 3.34 & 1.87 & $<10^{-4}$ & 0.65 \\
\hline
\end{tabular}

$N_{l}=$ Average number of minor events happening within the lifetime of the reactor; $N_{2}=$ Average number of moderate events happening within the lifetime of the reactor; $N_{3}=$ Average number of major events happening within the lifetime of the reactor; $N_{r}=$ Average number of times the reactor system needs to reduce its power output due to the external events; $N_{s}=$ Average number of times the reactor is shut down due to the external events; $N_{m}=$ Average number of times the core melts due the failed shutdown; $P=$ Probability that the reactor system is not affected by the external events.

The impact of the effectiveness of the mitigation measures adopted in the study on the resilience of the reactor system to the external event can also be analyzed. The response speed of the emergency fishing team is chosen for demonstration. By modifying the time for the emergency team to reach the fishing area to a new Normal distribution with the mean $(\mu)$ of 30 minutes and the standard deviation $(\sigma)$ of 5 minutes, the simulation is conducted with the probability defined for Set 1 .

From the simulation results computed, it is found that the average number of shutdowns due to the external events within the lifetime of the reactor $\left(N_{m}\right)$ is reduced to 1.45 while the number of the reduced performance $\left(N_{r}\right)$ times is increased to 2.71. It means that the new emergency team is able to reduce the number of the unscheduled reactor shutdown due to the external events, so that overall performance loss of the reactor system is decreased. However, it should be noted that its effectiveness is limited as the decrease in the number of reactor shutdowns is small. In addition, it is found the probability that the reactor system is not affected by the external events $(P)$ remained the same, which means the ability of the new measure for stopping the external events before they can affect the system performance is not improved.

\section{Conclusion}

In order to study of the resilience of nuclear power plants in response to climate change, the PN method is adopted in this paper. The abilities of the experimental reactor to absorb the impact of the possible intake structure blockage due to the outbreak of acetes, which is chosen as a case study of external events related to the climate change, and recovery from the accident are successfully assessed. It is found that such events do have significant impact on the resilience of reactor system.

The PN model constructed can easily be adapted to assess the impact of other external events related to climate change such as outbreak of other marine organisms, flooding, and so on. The impact of the availability, capability, and reliability of other safety systems on the system resilience will be investigated in the future. In addition, the effectiveness of different maintenance strategies, human factors and response actions will be investigated. This can readily emphasize the adaptability of the system for improving system resilience. It is deemed that the resilience evaluation methodology established in this paper can be used effectively in the design of future resilient NPP's to mitigate the effects of climate change.

\section{Acknowledgement}

The work was supported by the Engineering and Physical Sciences Research Council EPSRC [grant number EP/R021759/1], which is part of the UK-India Civil Nuclear Collaboration program.

\section{References}

BBC. 2016. Torness Nuclear Reactor Shut down over Seaweed Concern - BBC News. BBC. https://www.bbc.co.uk/news/uk-scotland-edinburgheast-fife-38070241.

Bian, X., C. Mou, Z. Yan, and J. Xu. 2009. Simulation Model and Fault Tree Analysis for AUV. In 2009 International Conference on Mechatronics and Automation, 4452-4457. Changchun: IEEE. http://ieeexplore.ieee.org/document/5246716/.

Edwards, R. 2013. Seaweed Shuts down Scottish Nuclear Reactor | Nuclear Power | The Guardian. The Guardian.

https://www.theguardian.com/environment/2013/nov /21/seaweed-torness-nuclear-reactor-east-lothian.

Fu, X., F. Du, X. Pu, X. Wang, and F. Han. 2020. Analysis on Critical Factors of Marine Organism Impacts on Water Intake Safety at Nuclear Power Plants. Journal of Nuclear Engineering and Radiation Science 6, no. $4 \quad$ (October 1): 1-6. https://asmedigitalcollection.asme.org/nuclearengine ering/article/doi/10.1115/1.4048112/1086215/Analys is-on-Critical-Factors-of-Marine-Organism.

IAEA. 1988. Component Reliability Data for Use in Probabilistic Safety Assessment, IAEA-TECDOC478. International Atomic Energy Agency. VIENNA: IAEA.

- 2003. Extreme External Events in the Design and Assessment of Nuclear Power Plants, IAEATECDOC-1341. Vienna: IAEA.

Oh, C.-W., and I.-J. Jeong. 2003. Reproduction and Population Dynamics of Acetes Chinensis (Decapoda: Sergestidae) on the Western Coast of Korea, Yellow Sea. Journal of Crustacean Biology 23, no. 4 (January 1): 827-835. https://academic.oup.com/jcb/articlelookup/doi/10.1651/C-2405.

Panteli, M., A.I. Nikolaidis, C.A. Charalambous, Y. Zhou, F.R. Wood, S. Glynn, and P. Mancarella. 2016. Analyzing the Resilience and Flexibility of Power Systems to Future Demand and Supply Scenarios. In 
2016 18th Mediterranean Electrotechnical Conference (MELECON), 1-6. IEEE. https://ieeexplore.iee.org/document/7495312.

Tang, Z., F. Cheng, X. Jin, L. Sun, R. Bao, and Y. Liu. 2017. An Automatic Marine-Organism Monitoring System for the Intake Water of the Nuclear Power Plant. Annals of Nuclear Energy 109 (November): 208-211. http://dx.doi.org/10.1016/j.anucene.2017.05.040.

Turnpenny, A., J. Coughlan, P. Crews, R. Bamber, and P. Rowles. 2010. Cooling Water Options for the New Generation of Nuclear Power Stations in the UK, SC070015/SR3.

http://publications.environmentagency.gov.uk.

USNRC. 2011. Service Water Problems. In General Electric Advanced Technology Manual. Nuclear Regulatory Commission. https://www.nrc.gov/docs/ML1414/ML14140A114. pdf.

Vaughan, A. 2018. In a Laver: Seaweed Shuts Nuclear Reactor Again in Bad Weather | EDF Energy | The Guardian.

https://www.theguardian.com/business/2018/mar/05/ seaweed-shuts-nuclear-reactor-edf-torness-plant.

Wang, J., and F. Duan. 2017. Risk Analysis of Water Intake Structure Blockage in a Nuclear Power Plant. In Proceedings of the 2017 25th International Conference on Nuclear Engineering, 1-8. Shanghai: ASME. 